\title{
Reactive Power Optimization of Distribution Network with Distributed Generators
}

\author{
Dongxin Chen ${ }^{1, a}$, Shuangyin Dai ${ }^{2, b}$, Huitan $\mathrm{Sh}^{3, \mathrm{c}}$, and Chaofeng Wei ${ }^{3, \mathrm{~d}}$ \\ ${ }^{1}$ State Grid Henan Electric Power Company , Zhengzhou 450001, China; \\ ${ }^{2}$ State Grid Henan Electric Power Research Institute , Zhengzhou 450001, China; \\ ${ }^{3}$ School of electrical engineering, Zhengzhou University, Zhengzhou 450001, China.

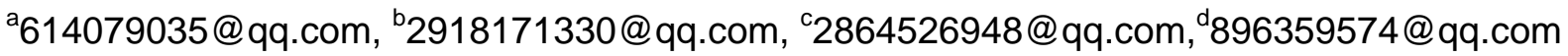

\begin{abstract}
Keywords:Distributed generators(DGs),sensitivity analysis,reactive power,bacteria colony optimization algorithm, distribution network
\end{abstract}

\begin{abstract}
Reactive power optimization of distribution network is necessary,because optimize configuration of distributed generators can effectively reduce power loss.A new method was proposed in this paper.Firstly, installation points of distributed power were found with the means of power grid loss sensitivity. On this basis, the bacteria colony optimization algorithm was used in the solution of reactive power optimization problem containing distributed generators of the distribution network.Finally, the simulation result was compared with the improved particle swarm and genetic algorithm, and verified the proposed algorithm has good practicability and adaptability with an example.
\end{abstract}

\section{Introduction}

Installation of Large-scale DGs bring new challenges to safe operation and optimal operation of the power system ${ }^{[1]}$. How to make full use of DG reactive dispatch ability to realize reactive power dispatch of power system was studied in this paper.The subject has important scientific and practical significance. [2,3] all use intelligent algorithm to select the site and capacity of the DGs of distribution network, without considering the reactive power optimization.Literature [4] combining genetic algorithm and simulated annealing algorithm to find the DG installation site with the system has the minimum loss.Bacteria colony optimization algorithm was applied in optimization of distribution network with DGs in literature [5], and compared its results with the improved PSO algorithm, and verified the superiority of the algorithm on the convergence speed and accuracy. Literature [6] proposed the method of network loss sensitivity based on linear analysis .

In view of the existing problems of reactive power optimization, a new method containing DGs for reactive power optimization of distribution network was proposed in this paper.

\section{Reactive power optimization model of the distribution network}

Based on the minimum of active power loss and highest node voltage qualification rate of the distribution network, the objective function and constraint conditions can be described as follows

(1) objective function.

$$
\mathrm{F}_{\text {min }}=P_{\text {loss }}+\sum_{i=1}^{N} \lambda_{\mu}\left(\frac{\Delta U_{i}}{U_{i \max }-U_{i \min }}\right)^{2}
$$

where $\mathrm{P}_{\text {loss }}$ is active network loss and $\mathrm{N}$ is system node; ${ }^{\lambda}$ is the punishment factor when the node

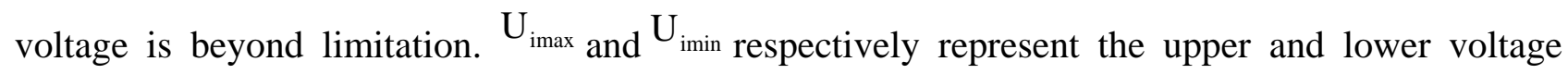
amplitude of nodes I and j. Where can be represented as

$$
\mathrm{P}_{\text {loss }}=\sum_{i=1}^{N} U_{i} \sum_{j \in i} U_{j}\left(G_{i j} \cos \theta_{i j}+B_{i j} \sin \theta_{i j}\right)
$$


where $\mathrm{U}_{\mathrm{i}}$ and $\mathrm{U}_{j}$ are the voltage amplitude of node I and $\mathrm{j} ; \mathrm{G}_{\mathrm{ij}}, \mathrm{B}_{\mathrm{ij}}$ and $\theta_{\mathrm{ij}}$ respectively represent the conductance,electrical susceptance and the voltage phase angle between nodes I and j.And

$$
\Delta \mathrm{U}_{\mathrm{i}}=\left\{\begin{array}{cc}
\mathrm{U}_{\mathrm{i}}-\mathrm{U}_{\mathrm{imax}} & \mathrm{U}_{\mathrm{i}}>U_{i \max } \\
0 & U_{i \min } \leq \mathrm{U}_{\mathrm{i}} \leq \mathrm{U}_{\mathrm{imax}} \\
\mathrm{U}_{\mathrm{imin}}-U_{i} & U_{i}<U_{i \min }
\end{array}\right.
$$

(2) constraint condition.The constraint condition of voltage and reactive power optimization includes two parts : equality constraint and inequality constraint.Equality constraint condition is the power balance equation of the node:

$$
\left\{\begin{array}{l}
\mathrm{P}_{\mathrm{i}}=\sum_{\mathrm{j}=1}^{N} e_{i}\left(G_{i j} e_{j}-B_{i j} f_{j}\right)+f_{j}\left(G_{i j} f_{j}+B_{i j} e_{j}\right) \\
\mathrm{Q}_{\mathrm{i}}=\sum_{\mathrm{j}=1}^{N} e_{i}\left(G_{i j} e_{j}-B_{i j} f_{j}\right)-f_{j}\left(G_{i j} f_{j}+B_{i j} e_{j}\right)
\end{array}\right.
$$

Inequality constraint conditions are each node's voltage not crossing the line voltage and the output limitation of reactive power compensation device.

Node voltage constraint:

$\mathrm{U}_{\text {imin }} \leq U_{i} \leq U_{i \max }$

Reactive compensation device output constraints:

$\mathrm{Q}_{\text {cimin }} \leq Q_{c i} \leq Q_{c i \max }$

Where $\mathrm{Q}_{\text {cimax }}$ and $\mathrm{Q}_{\text {cimin }}$ respectively represent the upper and lower constraint of the reactive compensation device.

\section{Selection of reactive power compensation based on network loss sensitivity of node}

Sensitivity analysis can directly response the relationship between the control variables and controlled variables. It mainly discuss the network loss sensitivity in this paper,namely the sensitive degree of system network loss when the active power $\mathrm{P}$ and reactive power $\mathrm{Q}$ of load nodes change .

Active network loss sensitivity can be represented as follows considering equation (2) and (4)

$$
\left\{\begin{array}{l}
\frac{\partial \mathrm{P}_{\text {loss }}}{\partial P_{l}}=\frac{\partial P_{\text {loss }}}{\partial \theta} \cdot \frac{\partial \theta}{\partial P_{l}}+\frac{\partial P_{\text {loss }}}{\partial U} \cdot \frac{\partial U}{\partial P_{l}} \\
\frac{\partial \mathrm{P}_{\text {loss }}}{\partial Q_{l}}=\frac{\partial P_{\text {loss }}}{\partial \theta} \cdot \frac{\partial \theta}{\partial Q_{l}}+\frac{\partial P_{\text {loss }}}{\partial U} \cdot \frac{\partial U}{\partial Q_{l}}
\end{array}\right.
$$

where $\mathrm{P}_{1}$ and $\mathrm{Q}_{1}$ respectively represent the input active power and reactive power of node $\mathrm{i}$ in distribution network.

$$
\left[\begin{array}{l}
\frac{\partial \mathrm{P}_{\text {loss }}}{\partial P_{l}} \\
\frac{\partial P_{\text {loss }}}{\partial Q_{l}}
\end{array}\right]=\left[\begin{array}{ll}
\frac{\partial P_{l}}{\partial U} & \frac{\partial Q_{l}}{\partial U} \\
\frac{\partial P_{l}}{\partial \theta} & \frac{\partial Q_{l}}{\partial \theta}
\end{array}\right]^{-1}\left[\begin{array}{l}
\frac{\partial P_{\text {loss }}}{\partial U} \\
\frac{\partial \mathrm{P}_{\text {loss }}}{\partial \theta}
\end{array}\right]
$$

It can get the sensitivity equations of active network loss of the system from equations (8) when reactive power change:

$$
\left[\partial \mathrm{P}_{\text {loss }} / \partial Q_{l}\right]=S\left[\begin{array}{l}
\partial P_{\text {loss }} / \partial \mathrm{U} \\
\partial P_{\text {loss }} / \partial \theta
\end{array}\right]
$$

where $\mathrm{S}$ is sensitivity matrix, and can be obtained from Jacobian matrix in power flow equation.

Node i can be represented as follows 


$$
\left\{\begin{array}{c}
\frac{\partial \mathrm{P}_{\text {loss }}}{\partial U_{i}}=2 \sum_{j=1}^{N} U_{j} G_{i j} \cos \theta_{i j} \\
\frac{\partial P_{\text {loss }}}{\partial \theta_{i}}=-2 U_{i} \sum_{j=1}^{N} U_{j} G_{i j} \sin \theta_{i j}
\end{array}\right.
$$

The node sensitivity factor can be calculated by equation(9)and(10).Select several nodes which coefficient is larger as the reactive power compensation nodes.

The steps finding DGs installation site by sensitivity can be described briefly as follows.

(1) Firstly, calculate the active network loss sensitivity for each node injection power of the system.

(2) Then order the nodes according to the value of sensitivity.

(3) Nodes which value of active network loss sensitivity is bigger are nodes waiting for selected to connect with DGs .

\section{Reactive power optimization based on bacterial colony algorithm}

1) Optimization algorithm based on bacterial colonies method Bacterial colony algorithm is a algorithm that simulate single bacteria and group colony growth process to find the optimal solution ${ }^{[7]}$. Describe the approximate process of the optimal solution in two ways: traveling and tumbling. Assume that the solution space of the optimization problem corresponding to the specified culture, the concentration of the nutrients in culture means the optimization degree of objective function optimization problem corresponding to individual bacteria. The pros and cons of each bacterium performance depends on the specified fitness of the objective function of problem being optimized.Growth of individual bacteria bound by nutrients and other environmental conditions. The largest scale of the population in the colonies is $\mathrm{N}$ in regulations optimization algorithm and the number of breeding of individual bacteria can't surpass $\mathrm{N}$.

After the adjustment period, absorb enough nutrients, bacteria begin to split.The bacterium will die if surpass the individual life cycle or meet the other regulative conditions of the death. Each bacteria has certain limits of perception, and can feel the experienced optimal location through the communication and exchange of information. Each bacteria constantly update themselves to change the position and velocity.Each bacteria must update their two properties in the process of each iteration calculation.Two properties of bacteria: traveling and tumbling. Traveling namely walk along the last direction of movement and move towards more and more favorable environment; rolling means to move towards other direction and get away from the bad environment. Bacteria will move to the optimal location point and achieve the target optimization through constant Traveling and tumbling.

The update formula of movement of traveling of individual bacteria are described as follows:

$$
\begin{aligned}
& \mathrm{V}_{k+1}=\alpha V_{\mathrm{k}}+r_{1} \text { rand } \cdot\left(f_{\text {best }}-x_{k}\right)+r_{2} \text { rand } \cdot\left(g_{\text {best }}-x_{k}\right) \\
& \mathrm{X}_{\mathrm{k}+1}=X_{k}+V_{k+1}
\end{aligned}
$$

where $V_{k}$ indicates the direction of movement of individual of the kth iteration, $X_{k}$ represents the position of the kth iteration, $f_{\text {best }}$ represents individual optimal location of the last, ${ }^{\text {best }}$ means the global optimal position now of the colonies, $\alpha, r_{1}$ and $r_{2}$ are coefficients, Rand is a random number on $(0,1)$.

When the value of objective function is not superior to the before, indicates bacteria individuals have not searched the nutrient enrichment zone. The agent of bacteria will use movement of tumbling to change the search direction and position. The Update formula is

$$
\mathrm{X}_{k+1}=X_{k}+R \text { rand } n
$$

where $\mathrm{R}$ is searching radius, Rand $\mathrm{n}$ is a random number on $(-1,1)$.

2) Implementation of bacterial colonies algorithm of reactive power optimization 
The steps to determine the installation capacity of DGs by using the bacterial colonies optimization algorithm are as follows.

(1) Initialize the system parameters of the distribution network, and then input the constraint conditions of control variables and state variables.

(2) Invoke the objective function of power flow calculation which satisfied the constraint conditions and adopt bacterial colony optimization algorithm to solve it.

(3) Initialize the parameters of the bacteria colonies algorithm .If these current individual objective function values is superior to the parent, perform step (5) after updating speed and position of the corresponding individual bacteria, or do step (4).

(4) Determine whether individual bacteria meets the death conditions.If yes,it means individual bacteria will die and return to (3),or directly back to step (5).

(5) Judge whether the number of bacteria species surpass the maximum population scale.If yet ,return to step (4),or just continue step (6).

(6) Judge the individual breeding conditions. If achieve individual bacteria breeding conditions,then return to step(3).

(7) Judge whether it meets the death conditions.If meets,jump out the iteration and output the result which means process end, otherwise return to step(3) according to the result of movement this time, and then update the parameters.

(8) Output the results.

\section{Analysis of example}

The example contain IEEE - 33 nodes system was analyzed in this study,Related programs were written by Matlab.The reference voltage of the system is $12.66 \mathrm{KV}$, benchmark capacity is $100 \mathrm{MVA}$, total load of system is 3.715 MW + j2.3 MVar, and the system has an on-load voltage regulating transformer and two sets of parallel compensation capacitor.The range of the ratio of on-load voltage regulating transformer is $0.9 \sim 0.9$. The capacity of two group parallel capacitor were set to $0.5 \mathrm{MVar}$. The largest scale of colony population was set to $S=20$, growth cycle of the individual bacteria was set to $\mathrm{N}=4$. Breeding conditions and condition of death were set to $\mathrm{N}_{\mathrm{P}=2} \mathrm{~N}_{\mathrm{r}}=2$. Other Parameter setting: $\alpha=0.7, r_{1}=r_{2}=1.5$ and the search radius $\mathrm{R}$ is set to $10^{-1}$.

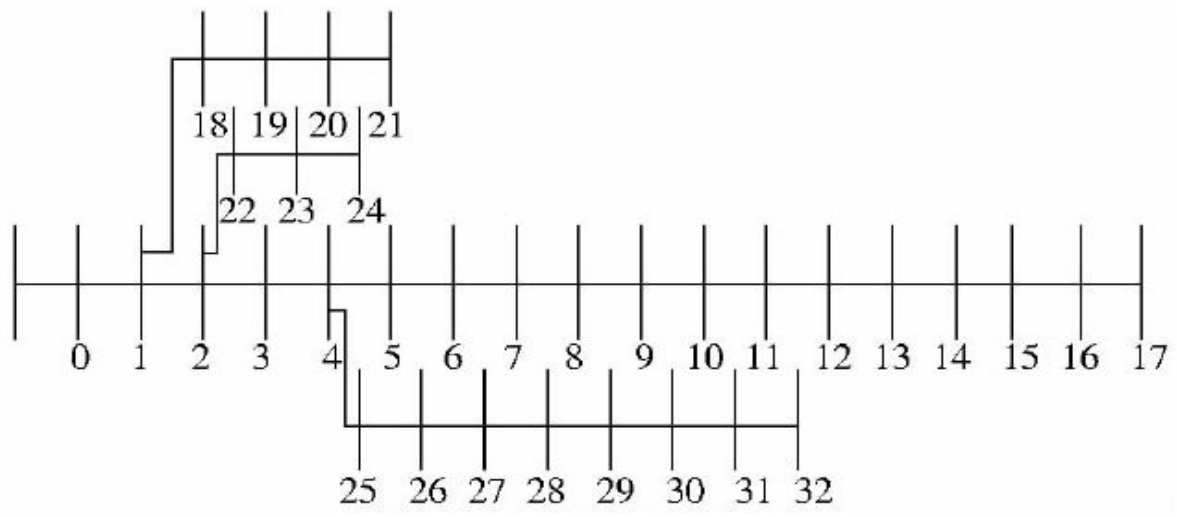

Fig.1 Diagram of the IEEE-33 nodes test system

Firstly, the sensitivity analysis method is used to calculate the sensitivity of active power and reactive power of the 33 nodes,it is just as shown in the Fig. 2 : 


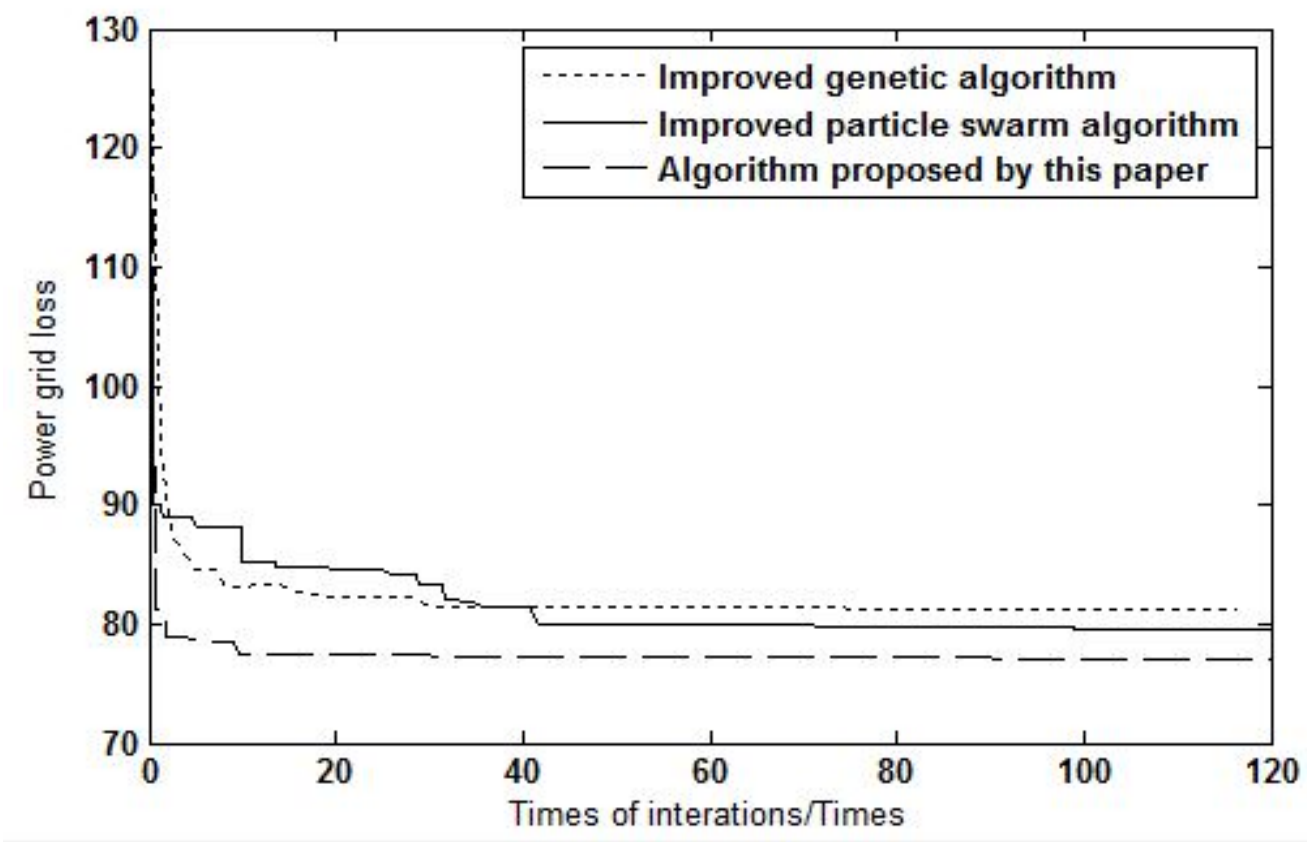

Fig.2 Figure of the power sensitivity of network nodes

It can be found that the 18th node have the largest sensitivity of active power network loss. So the candidate's installation position of the DGs is the 18th node. On this basis, bacteria colony algorithm, PSO algorithm and genetic algorithm were used respectively to calculate the installation capacity of DGs. The convergence curves of the three algorithms are shown in Fig.3

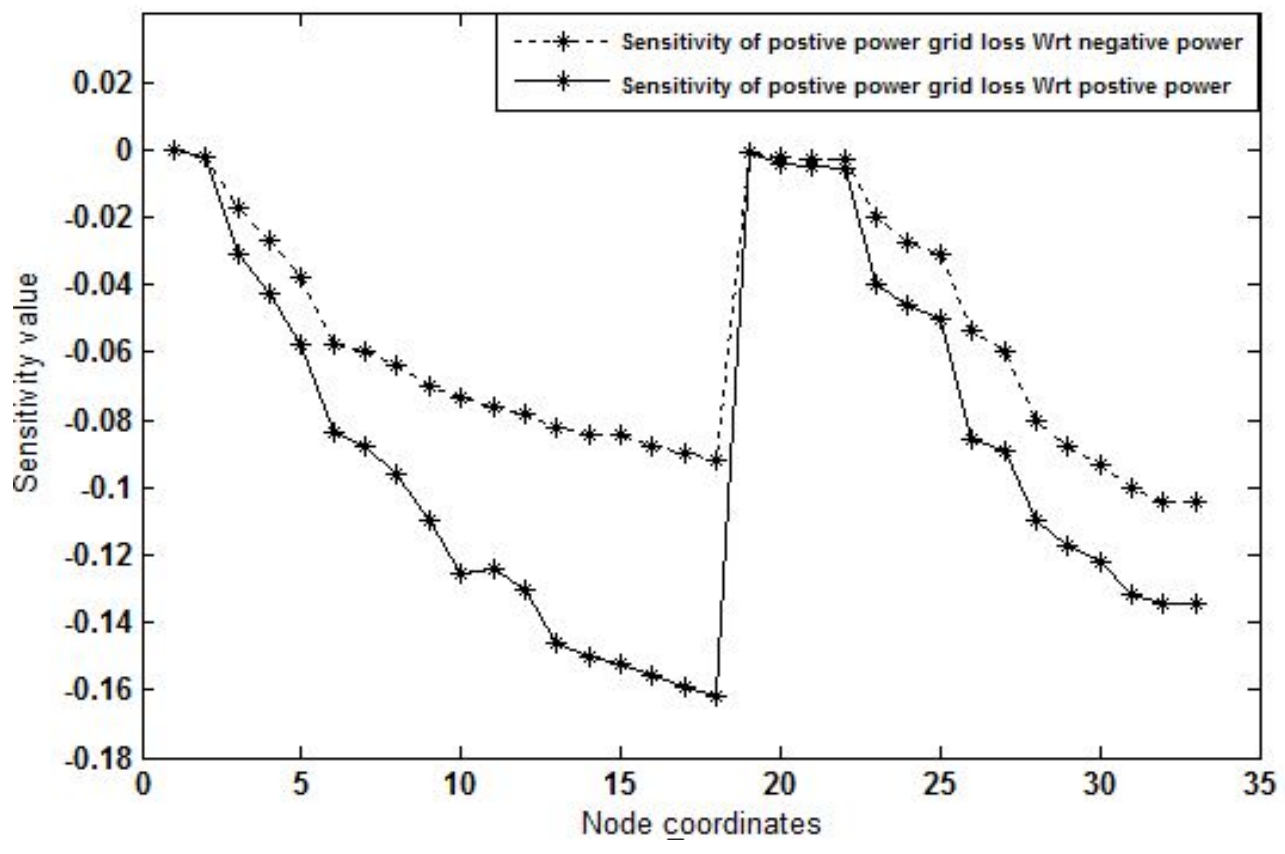

Fig.3 Graph of algorithm's convergence

It was found form the Fig. 3 that the algorithm in this study not only has a high convergence speed, but also can find the global optimal solution of high quality. In addition, the active network loss of the system fell from $130 \mathrm{KW}$ to $76.52 \mathrm{KW}$ after the access of DGs in distribution network,which reduce by $58.7 \%$. It proved that the reasonable planning of distributed power supply can reduce active network loss of the system . 


\section{Summary}

Reactive power optimization of distribution network system containing DGs was done in paper.It adopted bacterial colony optimization algorithm combining DGs installation site selection method based on sensitivity analysis and achieved good results in power system reactive power optimization. The result proved the necessity of reactive power compensation optimization of distribution network containing DG , as well as the feasibility and superiority of the improved algorithm.

\section{References}

[1] W. Pei and K. Li: Proceedings of the CSEE, Vol. 28 (2008) No.13,p.152.

[2] J.T. Zhang, H.Z. Cheng and L.Z. Yao: Proceedings of the CSEE, Vol. 29 (2009) No.16,p.1.

[3] D.Y. Ye, Z.Y. He and T.L. Zhang:Power System Technology, Vol. 35 (2011) No.6,p.155.

[4] Gandomkar.M, Vakilian.M and Ehsan.M: Proceeding of IEEE Canadian Conference on Electrical and Computer Engineering(2005).

[5] X.Z. Jian, Y. Li: China.Parent 201310140845(2013).

[6] Khatod.Dheeraj Kumar, Pant Vinay and Sharma Jaydev: IEEE Transactions on Power Delivery,Vol. 28 (2006) No.4,p.32.

[7] M. Li and C.W. Yang:Control Theory and Applications, Vol.28 (2011) No.2,p.223. 